Journal id: CCPQ_A_426719

Corresponding author: Judi Miller

Title: The effects of expectations on experiences with psychotherapy 


\title{
RESEARCH DIGEST \\ The effects of expectations on experiences with psychotherapy
}

\author{
Judi Miller* \\ University of Canterbury, Christchurch, New Zealand
}

5

The issue of how client expectations influence the outcome of psychotherapy has been a research interest for over 50 years with a number of authors in the 1950s, 60 s and 70s calling attention to the clinical importance of this variable (Frank, 1968). In most cases there is support for the view that pre-therapy expectations of clients that they are likely to benefit from psychotherapy influences the success of their treatment. A problem with most research, however, is that the evidence derives mainly from correlational self-report studies (Greenberg, Constantino, \& Bruce, 2005). A call has been made for systematic prior, or early, treatment expectancy enhancement strategies.

So, this issue, I am reporting on two studies that consider the client's perspective of their experience of expectations for their counselling. The first looks at the effect of expectations on client experiences, the second investigates the client's experience of the expectation of change in therapy.

(1) Seligman, L.D., Wuyek, L.A., Geers, A.L., Hovey, J.D., \& Motley, R.L. (2009). The effects of inaccurate expectations on experiences with psychotherapy. Cognitive Therapy Research, 33, 139-149.

This research involves two studies; one to explore whether client expectations are consistent with cognitive behavioral therapy or other approaches, the second to examine whether client expectations about treatment affect perceptions of treatment. While these aims may seem too narrow (i.e., focused on cognitive behavioral therapy as a preferred therapy) for a general readership, I think the studies provide opportunities for reflection on our practice, regardless of the therapeutic modality. The first such point occurs in the research introduction where the authors explore the origins of expectations. They suggest that most clients' expectations come from cultural avenues such as television, films and literature, and they claim that these avenues reference non-directive and psychodynamic types of therapeutic interventions. While this claim is supported by evidence in the study, and by most cartoons in which therapy is depicted, I suggest that there are exceptions including television shows such as $\mathrm{Dr}$ Phil where cognitive behavior therapy is prominent. Nevertheless, the idea that client expectations of counselling and therapy are influenced by depictions in entertainment media is important to acknowledge and address in initial therapy sessions.

This research does have limitations, the most important of which is the use of a sample of mainly Caucasian undergraduate psychology female students who are not "real" clients. Despite this limitation, the paper describes a tidy, empirical study

\footnotetext{
*Email: judi.miller@canterbury.ac.nz
} 
where these students were asked about their perceptions of what a therapist does, what a client expects and what happens in therapy. In each case, $95 \%$ of the students responded with answers that described approaches and techniques not typical of cognitive behavioral therapy.

In the second research study, students participated in a screening session and agreed to take part in a treatment phase. They were shown a short video of either cognitive behavioral therapy or a non-directive approach before completing a questionnaire in which they predicted the therapy's helpfulness, pleasantness and enjoyability. These dimensions are not those normally used as evaluative constructs of efficacy and successful outcome and I am surprised that the researchers did not comment on this. Participating students then chose a non-clinical academic problem, e.g., procrastination, and were randomly assigned to a treatment condition (three sessions of either cognitive behavioral therapy or nondirective therapy). After each therapy session, participants completed the same questionnaire and 1-2 weeks after all therapy sessions, they were given a post-treatment interview. Ethical considerations about client safety were well addressed.

Results demonstrated that, upon viewing the initial videos, there was not a significant difference in affective reactions to cognitive behavioral therapy and nondirective approaches. Notable, however, was the finding that participants who had been expecting nondirective therapy (because they had viewed a nondirective video) and had received cognitive behavioral therapy viewed their first session as less helpful than they expected. Those who viewed and received nondirective therapy viewed the first session as more helpful than originally expected. This pattern was reversed for participants expecting cognitive behavioral therapy; the cognitive behavioral therapy session was considered more helpful than the nondirective. These patterns were present in participant perceptions of pleasantness and enjoyability. Furthermore, these patterns remained constant over three therapy sessions with participants reacting more positively when they received treatment that was consistent with their expectation.

While these results may appear unsurprising, it is helpful to find some empirical research, despite it limitations, that attempts to provide evidence in support of some of the common factors research (Lambert, 2007). It is also useful to find more evidence to support the view that expectations upon entering psychotherapy do affect client affective reactions which may influence their motivation for engaging in treatment.

The authors suggest that cognitive behavioral therapists should use accounts from former clients to induct their clients about what to expect in their therapy sessions. I would suggest that all clients should be informed about any therapeutic approach prior to their first session (see Manthei \& Miller, 2000)

\section{References}

Frank, J.D. (1968). The influence of patients' and therapists' expectations on the outcome of psychotherapy. British Journal of Medical Psychology, 41, 349-356.

Greenberg, R.P., Constantino, M.J., \& Bruce, N. (2005). Are patient expectations still relevant for psychotherapy process and outcome? Clinical Psychology Review, 26(8), $657-678$. 
Lambert, M. (2007). Presidential Address: What have we learned from a decade of research aimed at improving psychotherapy outcome in routine care. Psychotherapy Research, $17(1), 1-14$.

Manthei, R., \& Miller, J. (2000). Good counselling: A guide for clients. Auckland: Pearson.

(2) Carey, T.A., Carey, M., Stalker, K., Mullan, R.J., Murray, L.K., \& Spratt, M.B. (2007). Psychological change from the inside looking out: A qualitative investigation. Counselling and Psychotherapy Research, 7(3), 178-187.

This research seeks to address concerns that, while client change during psychotherapy is an expectation, the multiplicity of definitions of change has been ignored and the client's role in the change process has been under-emphasized (Duncan \& Miller, 2000). Furthermore, the authors wanted to explore how change actually happens. While the research reports interesting and helpful findings, it also highlights the difficulties inherent in this type of research. The researchers used a sampling grid and attempted to recruit equal numbers of male and female clients representing a wide age range but, in the end, their participants were mainly women aged over 30 years. They succeeded in having participants whose initial presenting problems were diverse. Four clinical psychologists, a counselling psychologist and two cognitive behavior therapists recruited participants to the study. Two hundred and seven clients were invited to participate, 47 agreed to participate by attending a median number of 6 sessions, and 27 of these participants took part a posttreatment interview. This interview provided the data for the study.

During the recorded interview, a topic guide was used to ask participants to describe what they understood change to be and how it happened (or did not) during their therapy. Ethical considerations and the method of data collection and analysis are clearly described in this article. Of the participants interviewed, two experienced change before they attended their first interview, three did not experience change during the course of therapy and the remaining 22 experienced change during the course of therapy. When asked, participants were generally unable to define change. Rather, they responded by describing their experiences of change in three domains: feelings, thoughts and actions. Evidence, in the form of clients' interview excerpts supported the view that change involved a shift from negative to positive experiences.

While much of the content of this article supports other research on the nature and process of change, there are some distinct contrasts. The major difference is the way in which clients described change as both a gradual process and an identifiable moment, rather that a series of stages. The second difference is that these descriptions came from the people experiencing the change rather than other sources and they were not guided by assumptions of a particular change model. By using client voices the authors have highlighted clients' views about how change occurs with comments such as "the light going on", "a load being lifted" and "like steam out of a kettle". Contributing factors included readiness and motivation, perceived personal characteristics, tools and strategies, learning and insight, interaction with therapist and relief of talking. Interestingly, most attributed the change to their own hard work. The most important factor for people who experienced no change was their perceived sense of self - e.g., resistant or perfectionist. I was disappointed that no mention was made of comments made by clients who experienced change before 
135 they attended their first interview as this is an expectation of some therapeutic models, for example solution-focused brief therapy.

The authors conclude that there are two main implications that might arise from this study. The first arises from most clients' descriptions of the change process as a period of negativity, then a sudden change moment followed by a protracted period of positivity. The implication taken from this by the authors is that it might be important for psychological treatments to facilitate progress towards an impasse and then to support the person while they experience this impasse and develop insights to resolve it rather than trying to avoid it. The second implication arises from the rich data provided by clients. It behoves us to listen to those who are undergoing change in order to understand this process more accurately.

I suggest that these two articles provide opportunities to continue to think about the importance of expectations in our work. Both indicate that an expectancyenhancement strategy should be to collaboratively explore client and counsellor expectations at the outset of therapy.

\section{Reference}

Duncan, B.L., \& Miller, S.D. (2000). The client's theory of change: Consulting the client in the integrative process. Journal of Psychotherapy Integration, 10, 169-187. 